\title{
Aumento de transaminasas: una manifestación de distrofia muscular de Duchenne
}

\author{
MARÍA DE LOS ÁNGELES AVARIA ${ }^{1,3}$, MARÍA DE LOS ÁNGELES BEYTÍA ${ }^{3}$, \\ KARIN KLEINSTEUBER ${ }^{3,4}$, ELIANA RODILLO ${ }^{4}$, SYLVIA ALEGRÍA ${ }^{2,3}$ \\ 1. Unidad Neurología Pediátrica. Hospital de Niños Dr. Roberto del Río. \\ 2. Unidad Gastroenterología Infantil. Clínica Las Condes. \\ 3. Departamento de Pediatría y Cirugía Infantil Campus Norte Universidad de Chile. \\ 4. Unidad de Neurología Infantil, Clínica Las Condes. \\ Santiago, Chile.
}

\begin{abstract}
Transaminases increase: a manifestation of Duchenne's muscular dystrophy

Commonly used in clinical practice, glutamic oxalacetic (GOT) and glutamic piruvic (GPT) transaminases are produced in various body tissues, including striated muscle, so their blood elevation is not due exclusively to liver disease. The objective of this study is to demonstrate the correlation between elevated creatinkinase (CK) and transaminases in patients with diagnosis of Duchenne muscular dystrophy (DMD), the most frequent neuromuscular disease in children. Patients and Method: Assessment in 61 children with diagnosis of DMD of CK, AST and ALT levels, and their correlation. Results: All patients had increase of CK $(\overline{\mathrm{x}}=13.363 \mathrm{IU} / \mathrm{L})$, AST $(\overline{\mathrm{x}}=203 \mathrm{IU} / \mathrm{L})$ and ALT $(\overline{\mathrm{x}}=194 \mathrm{IU} / \mathrm{L})$ above normal values. The increase of transaminases related directly with the increase of CK. Conclusion: Patients with DMD have increased transaminases, so it is necessary to include this diagnostic possibility in a child with hypertransaminemia, prior to performing liver biopsy. (Key words: Duchenne, muscular dystrophy, transaminases, creatinkinase).

Rev Chil Pediatr 2012; 83 (3): 258-261
\end{abstract}

\section{RESUMEN}

Las transaminasas que comúnmente se utilizan en clínica, glutámico oxalacética (GOT) y glutámico pirúvica (GPT) son producidas en varios tejidos del organismo entre los cuales se cuenta el músculo estriado, por lo que la elevación de transaminasas en sangre no es producida exclusivamente por enfermedades hepáticas. Objetivo: Demostrar la correlación entre el alza de la creatinkinasa (CK) y transaminasas en pacientes con el diagnóstico de distrofia muscular de Duchenne (DMD), la enfermedad neuromuscular más frecuente en niños. Pacientes y Método: Evaluación en 61 niños con diagnóstico de DMD de los niveles de CK, GOT y GPT y la relación entre ellos. Resultados: Todos los pacientes presentaron aumento de CK ( $\overline{\mathrm{x}}=13.363 \mathrm{IU} / \mathrm{L})$, GOT $(\overline{\mathrm{x}}=203 \mathrm{IU} / \mathrm{L})$ y GPT $(\overline{\mathrm{x}}=194 \mathrm{IU} / \mathrm{L})$ sobre los valores normales. El aumento de transaminasas se relacionó en forma directa con aumento de CK. Conclusiones: Los pacientes con DMD presentan transaminasas aumentadas, por lo que es necesario incluir esta posibilidad diagnóstica en niños con hipertransaminasemia, previo a realizar biopsia hepática.

(Palabras clave: Duchenne, distrofia muscular, transaminasas, creatinkinasa).

Rev Chil Pediatr 2012; 83 (3): 258-261

Trabajo recibido el 03 de enero de 2012, devuelto para corregir el 30 de enero de 2012, segunda versión el 12 de marzo de 2012, aceptado para publicación el 02 de abril de 2012.

Correspondencia a:

María de los Ángeles Avaria

E-mailmaavaria@gmail.com 


\section{Introducción}

La Distrofia Muscular de Duchenne (DMD) es una de las afecciones neuromusculares más frecuentes en la infancia. Presenta herencia recesiva ligada al cromosoma $\mathrm{X}$, es heredada en un $70 \%$ de los casos y afecta a alrededor de 1 en 3500 recién nacidos del sexo masculino ${ }^{1}$. El gen afectado, ubicado en la región Xp21, es responsable de la producción de la proteína Distrofina. Deleciones, mutaciones puntuales y duplicaciones en el gen, dan origen a una proteína truncada, que es rápidamente degradada, con la consiguiente alteración de todo el complejo de proteínas asociada a ella ${ }^{2}$. Se presenta con debilidad predominantemente proximal, que se manifiesta con dificultad o retraso de la marcha y caídas frecuentes. La debilidad es progresiva y la evolución natural conduce a la pérdida de la marcha antes de los 13 años. El diagnóstico precoz tiene importantes implicancias para el paciente, ya que el tratamiento corticoidal ha demostrado lentificar la progresión de la enfermedad ${ }^{3}$ y la terapia física iniciada a edades tempranas permite minimizar las contracturas que dificultan la movilidad. Para la familia permite brindar una asesoría genética oportuna. Sin embargo el diagnóstico se retrasa por consulta tardía y errores en la interpretación de las características clínicas que presenta el paciente ${ }^{4,5}$, incluyendo en algunos casos el no considerar, en un paciente asintomático, el alza de transaminasas como producto de enfermedad muscular, lo que puede corroborarse con un examen sencillo como el aumento de niveles séricos de Creatinkinasa, un sensible indicador de daño muscular.

El objetivo de este trabajo es evaluar la correlación entre el alza de la creatinkinasa $(\mathrm{CK})$ $\mathrm{y}$ transaminasas en pacientes con diagnóstico de distrofia muscular de Duchenne (DMD).

\section{Pacientes y Método}

La medición de enzimas séricas creatinkinasa (CK) y transaminasas (GPT y GOT) es parte de la evaluación de rutina en pacientes con distrofia muscular en nuestro centro. En 61 niños evaluados por los autoras entre los años 1999 y 2011, controlados en el policlí- nico de neurología del Hospital Roberto del Río y de la consulta privada, con diagnóstico de distrofia muscular de Duchenne confirmado mediante biopsia muscular con ausencia de la proteína Distrofina y/o análisis de genética molecular concordante con el diagnóstico clínico, se analizaron los niveles de las enzimas creatinkinasa (CK) y transaminasas (GPT y GOT) y la correlación entre ellas.

Se les aplicó el coeficiente de relación de Pearson mediante programa SMSS para evaluar si existe relación lineal entre los valores de GOT y GPT con los valores de Creatinkinasa en forma independiente.

Se aplicó la prueba t-student para los valores de GOT y GPT para determinar si existían diferencias significativas entre los valores de ambas transaminasas.

Este estudio fue aprobado por el Comité de Ética de la Investigación del Servicio de Salud Metropolitano Norte.

\section{Resultados}

El 100\% de los casos mostró aumento de Creatinkinasa y transaminasas GOT y GPT sobre los valores normales. El promedio de Creatinkinasa fue de 60,7 veces el valor normal, el promedio de GOT fue 5,4 veces el valor normal y el de GPT 4,7 veces el valor normal (tabla 1).

Evaluado con la prueba de t-student las diferencias entre ambas transaminasas GOT y GPT no son significativas ( $\mathrm{p} \leq 0,05$, Intervalo de Confianza de 95\%). Los resultados El coeficiente de correlación entre CK y GOT fue $=0,273(\mathrm{p} \leq 0,05)$, y entre CK y GPT $=0,302$ $(\mathrm{p} \leq 0,05)$.

\section{Tabla 1. Valores promedio y de referencia de} enzimas Creatinkinasa y Transaminasas

\begin{tabular}{|crrr|}
\hline $\mathbf{n}=\mathbf{6 1}$ & \multicolumn{1}{c}{ Valor promedio } & $\begin{array}{c}\text { Valor } \\
\text { referencia }\end{array}$ \\
CK & 13363 & $(4170-69710) \mathrm{IU} / \mathrm{L}$ & $\leq 220 \mathrm{IU} / \mathrm{L}$ \\
GOT & 203 & $(53-642) \mathrm{IU} / \mathrm{L}$ & $\leq 37 \mathrm{IU} / \mathrm{L}$ \\
GPT & 194 & $(76-898) \mathrm{IU} / \mathrm{L}$ & $\leq 41 \mathrm{IU} / \mathrm{L}$ \\
\hline
\end{tabular}

CK: creatinkinasa. GOT: Transaminasa glutámico oxalacética. GPT: Transaminasa glutámico pirúvica. 


\section{Discusión}

Esta es la primera serie de mayor tamaño que reafirma lo mencionado por Sibley en 1949 , en relación a que la aspartato-aminotransferasa y la alanino-aminotransferasa se encuentran no sólo en el hígado, sino también en otros tejidos, entre ellos el músculo esquelético $^{6}$. Posteriormente se describe la presencia de estas enzimas en hígado, riñones y músculo ${ }^{7-9}$, pero frente a una hipertransaminemia, los médicos aún mantienen el foco en el estudio hepático, ignorando la posibilidad de otras etiologías. Estudios en adultos asintomáticos con elevación de transaminasas señalan mayoritariamente las causas hepáticas como etiologías probables, recomendando la biopsia hepática. Sin embargo, se ha reportado que un 7\% corresponde a causas extrahepáticas como la enfermedad celíaca, miopatías y ejercicio excesivo, lo cual es probablemente más frecuente en niños ${ }^{10-12}$. Se han reportado pequeñas series de casos de distrofia muscular de Duchenne con hipertransaminemia, pero nuestra serie permite concluir que el aumento de ambas transaminasas es lo habitual en la DMD y que su aumento se relaciona en forma directa con la Creatinkinasa total, enzima ya aceptada como parte del diagnóstico y siempre aumentada en los pacientes DMD.

La aspartato aminotransferasa (AST o GOT) y alanina aminotransferasa (ALT o GPT) son enzimas intracelulares que catalizan el aspartato y la alanina a glutamina y acetil CoA respectivamente, los cuales son utilizados en el ciclo de Krebs para la producción de energía. La GOT se encuentra en mayores concentraciones en el tejido miocárdico, seguido del músculo esquelético, hígado y riñones. En cambio, GPT muestra mayores concentraciones en hígado y riñones, con bajos niveles en músculo esquelético ${ }^{13}$. De acuerdo a ésto, se esperaba encontrar niveles mayores de GOT que de GPT, sin embargo, no encontramos diferencias significativas entre estas enzimas $(p \leq 0,05)$. Estudios anteriores mencionan esta discordancia ${ }^{14}$. Los hallazgos podrían explicarse por otros factores determinantes en la concentración plasmática, como el catabolismo enzimático y su eliminación. Estudios en animales muestran que la eliminación renal de GPT es más lenta y que el tiempo de vida media de ésta es 42 horas, en cambio de GOT es de 17 horas $^{15}$, lo que podría explicar en cierta medida nuestros resultados.

El aumento de transaminasas GOT y GPT en distrofias musculares se ha comunicado en la literatura incluso en el período asintomático de la enfermedad, como casos únicos o series pequeñas ${ }^{16-22}$.

No todas las enfermedades neuromusculares, ni todas las distrofias presentan aumento de CK, pero otras patologías musculares aparte de la DMD, que cursan con aumento de CK, también se asocian a aumento de transaminasas. Dentro de éstas se incluyen las miopatías inflamatorias, glicogenosis con compromiso muscular, sarcoglicanopatías y otras distrofias musculares ${ }^{23}$.

\section{Conclusión}

1. Los niños con Distrofia muscular de Duchenne presentan aumento de la aspartatoaminotransferasa (GOT) y alanino-aminotransferasa (GPT), enzimas principalmente hepáticas, pero también presentes en el músculo esquelético.

2. El aumento de ambas transaminasas se relaciona en forma directa con el aumento de Creatinkinasa.

3. Reconocer la distrofia muscular como causa de hipertransaminemia tiene consecuencias inmediatas de extrema importancia: evitar realizar biopsias hepáticas innecesarias y hacer un diagnóstico precoz de distrofia muscular para iniciar el tratamiento adecuado.

4. Los pediatras deben llevar a cabo un examen físico completo de estos pacientes, buscando en particular signos sutiles como hipertrofia de pantorrillas y debilidad muscular de las extremidades inferiores y de cuello.

Se recomienda solicitar estudio de Creatinkinasa, previo a considerar la biopsia hepática en los niños con hipertransaminemia, ya que en 5 pacientes de nuestra muestra el diagnóstico inicial fue de hepatitis. 


\section{Agradecimientos}

Al Sr. Profesor Pablo Beytia Reyes, magíster en Sociología de la Pontificia Universidad Católica de Chile por su colaboración en el análisis estadístico de los datos.

\section{Referencias}

1.- Escolar D, Leshner R: Muscular Dystrophies. Pediatric Neurology. Principles and Practice. Swaiman, Ashwal, Ferriero(eds). 4th ed. 2006; 1969-87.

2.- Hoffman EP, Brown RH Jr, Kunkel LM: Dystrophin: The protein product of the Duchenne muscular dystrophy locus. Cell 1987; 51 (6): 919-28.

3.- Moxley RT 3rd, Ashwal S, Pandya S, et al: Practice parameter: Corticosteroid treatment of Duchenne dystrophy: report of the Quality Standards Subcommittee of the American Academy of Neurology and the Practice Committee of the Child Neurology Society. Neurology 2005; 64: 13-20.

4.- Avaria M, Kleinsteuber K, Herrera L, Carvallo P: Tardanza en el diagnóstico de la distrofia muscular de Duchenne en Chile. Rev Med Chile 1999; 127: 65-70.

5.- Ciafaloni E, Fox DJ, Pandya S, et al: Delayed diagnosis in Duchenne muscular dystrophy: data from the Muscular Dystrophy Surveillance, Tracking, and Research Network (MD STARnet). J Pediatrics 2009; 155 (3): $380-85$

6.- Sibley JA, Lehninger AL: Aldolase in the serum and tissues of tumor-bearing animals. J Natl Cancer Inst 1949; 9: 303-9.

7.- Thomson W, Leyburn P, Walton J: Serum enzyme activity in muscular dystrophy. BMJ 1960; 2: 1276-81.

8.- Wroblewski F: The clinical significance of transaminase activities of serum. Am J Med 1959; 27: 911-9.

9.- Pearson CM: Serum enzymes in muscular dystrophy and certain other muscular and neuromuscular diseases. N Eng J Med 1957; 256: 1069-75.

10.- Daniel S, Ben-Menachem T, Vasudevan G, Ma CK, Blumenkehl M: Prospective evaluation of unexplained chronic liver transaminase abnormalities in asymptomatic and symptomatic patients. Am J Gastroenterol 1999; 94: 3010-4.

11.- Pratt D, Kaplan M: Evaluation of abnormal liver- enzyme results in asymptomatic patients. N Eng J Med 2000; 342: 1266-71

12.- Limdi JK, Hyde GM: Evaluation of abnormal liver function tests. Postgrad Med J 2003; 79: 307-12.

13.- Henry JB: Clinical Diagnosis and Management by Laboratory Methods. Philadelphia, PA, W. B. Saunders, 1991: 248-84.

14.- Kohli R, Harris D, Whitington P: Relative Elevations of serum Alanine and Aspartate Aminotransferase in Muscular Dystrophy. J Pediatr Gastroenterol Nutr 2005; 41 (1): 121-4.

15.- Kasper DL, Fauci AS, Longo DL, Braunwald E: Myocardial Infarction. In Harrinson Principles of Internal Medicine. Kasper D, Braunwald E, Fauci AS, Hauser S, Longo DL, Jameson JL.(Eds) 16th ed, Mac Graw Hill, $2005 ; 1450$.

16.- Rutledge J, Andersen J, Fink CW, Strickland A: Persistent hypertransaminasemia as the presenting finding of childhood muscle disease. Clin Pediatr (Phila) 1985; 24: 500-3

17.- Schwarz KB, Burris GC, De Mello DE, et al: Prolonged elevation of transaminase concentration in children with unsuspected myopathy. Am J Dis Child 1984; 138: 1121-4.

18.- Tim RW, Gaskell P, Stajich J: A "liver test" is not a liver test. Measuring serum creatine kinase can identify muscular dystrophy and avoid liver biopsy. N C Med J 1998; 59: 238-41.

19.- Korones DN, Brown MR, Palis J: "Liver function tests" are not always tests of liver function. Am J Hematol 2001; 66: 46-8.

20.- Morse RP, Rosman NP: Diagnosis of occult muscular dystrophy: importance of the "chance" finding of elevated serum aminotransferase activities. J Pediatr 1993; 122: 254-6.

21.- Stein MT, Tipnis NA, Schultz P: Fatigue, decreased interest in play, motor delay, and elevated liver function tests in a 4-year-old boy. J Dev Behav Pediatr 2002; 23 : $37-41$

22.- Zamora S, Adams C, Butzner JD, Machida H, Scott $R B$ : Elevated aminotransferase activity as an indication of muscular dystrophy: Case reports and review of the literature. Can J Gastroenterol 1996; 10: 389-93.

23.- Urganci N, Erkan T, Serdaroglu P, Oztelik G, Dogan $S$, Kayaalp $N$ : A rare cause of high transaminasemia: autosomal muscle dystrophy with gamma sarcoglycan. J Pediatr Gastroenterol Nutr 2001; 32 (3): 327-9. 